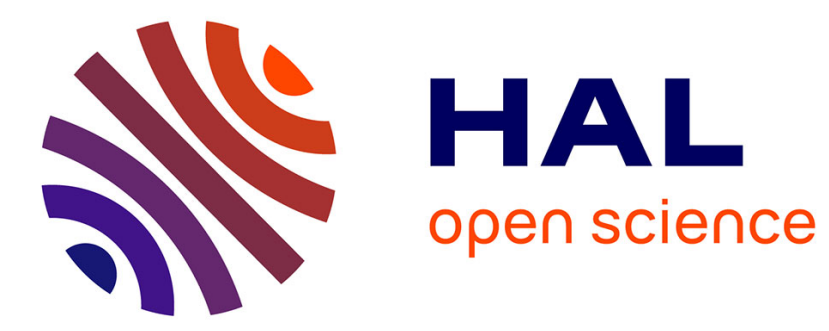

\title{
Travailler avec les catalogues d'éditeurs
}

Portail Persée, Perséides Data, Persée Blog, Marie-Christine Vinson

\section{To cite this version:}

Portail Persée, Perséides Data, Persée Blog, Marie-Christine Vinson. Travailler avec les catalogues d'éditeurs. Pratiques: linguistique, littérature, didactique, 1995, 88, pp.37-50. hal-03207069

\section{HAL Id: hal-03207069 \\ https://hal.univ-lorraine.fr/hal-03207069}

Submitted on 23 Apr 2021

HAL is a multi-disciplinary open access archive for the deposit and dissemination of scientific research documents, whether they are published or not. The documents may come from teaching and research institutions in France or abroad, or from public or private research centers.
L'archive ouverte pluridisciplinaire HAL, est destinée au dépôt et à la diffusion de documents scientifiques de niveau recherche, publiés ou non, émanant des établissements d'enseignement et de recherche français ou étrangers, des laboratoires publics ou privés.

\section{(이) $\$$}

Distributed under a Creative Commons Attribution - NonCommercial - NoDerivatives| 4.0 


\section{Travailler avec les catalogues d'éditeurs}

\section{Marie-Christine Vinson}

\section{Citer ce document / Cite this document :}

Vinson Marie-Christine. Travailler avec les catalogues d'éditeurs. In: Pratiques : linguistique, littérature, didactique, ${ }^{\circ} 88$, 1995. pp. 37-50;

doi : https://doi.org/10.3406/prati.1995.1758

https://www.persee.fr/doc/prati_0338-2389_1995_num_88_1_1758

Fichier pdf généré le 13/07/2018 


\title{
TRAVAILLER AVEC LES CATALOGUES D'ÉDITEURS POUR LA JEUNESSE
}

\author{
Marie-Christine VINSON
}

La littérature de jeunesse se distingue de moins en moins par ses éditions. Elle a par contre ses collections spécifiques et s'offre aux jeunes lecteurs comme un monde multiple et divers dans lequel il faut savoir se repérer.

Si le vrai lecteur, le lecteur autonome est celui qui sait élaborer des choix, des parcours de lecture et aller ainsi au bout de ses envies ou de ses besoins, il faut en général aider les élèves à se construire les compétences culturelles nécessaires à l'exercice de cette autonomie.

Se pose alors à l'enseignant la question de la situation de travail à mettre en place dans sa classe pour atteindre cet objectif. Nous proposons ici une possibilité de réponse didactique qui introduit un outil particulier, le catalogue d'éditeurs, et qui s'appuie sur la mise en place de sociabilités liseuses chez les enfants.

\section{Le catalogue d'éditeurs}

Le catalogue informe de la production d'un éditeur. Certaines maisons publient plusieurs catalogues comme par exemple l'École des loisirs qui décline ainsi ses différentes collections à travers des supports très diversifiés. D'autres comme L'Harmattan ou les Editions Nord-Sud ne proposent qu'un seul catalogue. De toute façon chaque éditeur sortant sa plaquette, il est tout à fait possible de se constituer un fonds riche et varié, représentatif de la production actuelle.

Le travail dont il est question ici écarte volontairement les sélections proposées par les organismes spécialisés (Revue des livres pour enfants, CRILJ...) qui s'adressent plutôt aux adultes prescripteurs de lecture (enseignants, bibliothécaires...). Bien évidemment le destinataire des catalogues n'est pas sans ambiguïté : il faut à la fois convaincre le jeune lecteur mais aussi l'adulteacheteur à qui appartient dans de nombreux cas la décision finale. 
L'intérêt du catalogue réside dans son statut et dans sa fonction. Ni livre-objet insolite et déroutant, ni objet-livre exactement semblable aux autres livres, il se définit plutôt comme un objet du livre, un petit livre sur les livres, un méta-livre dont le rôle est d'instaurer une médiation entre le texte et son lecteur. Intermédiaire de lecture mis à disposition chez les libraires, gratuit, consultable sur place ou à emporter chez soi, sa manipulation et sa pratique font partie des gestes qui distinguent le lecteur autonome et expert. Faire entrer ce nouveau support dans la classe place le jeune lecteur en situation de retravailler et repenser son rapport au livre, le confronte à l'abondance et à l'actualité des livres autrement que ne le font le C.D.I. et la bibliothèque.

\section{Les sociabilités liseuses}

Le travail sur les catalogues permet également de faire jouer les sociabilités entre élèves, les échanges et les partages de lecture. Ainsi la classe peut devenir un marché de lecture où vont circuler des discours sur les livres : discours sur les livres que tiennent les catalogues eux-mêmes mais aussi discours que les élèves seront amenés à élaborer sur leur propre lecture à destination des pairs pour faire connaître leur avis de lecteur.

Nous avons montré dans un précédent numéro de Pratiques ( $n^{\circ} 80$, "Pratiques de lecteurs ", décembre 1993) l'importance de ces réseaux de sociabilités qui participent de la valeur donnée à la lecture. Comment en effet se construire de réels comportements de lecteur si jamais ne s'offrent à soi des situations où l'on puisse mettre en pratique les savoirs et les savoir-faire acquis sur les livres, si jamais ne se présente l'occasion de réaffirmer dans la sécurité communautaire du groupe de pairs-lecteurs la croyance en l'importance de la lecture ? Les chances de lire augmentent si l'on fréquente des gens qui savent conseiller, parler le livre, le prêter. Autant dire que l'enseignant a tout intérêt à travailler à la construction de réseaux de sociabilités où les élèves puissent apprendre à formuler des discours sur leur lecture et à les échanger dans le cadre de situations de communication fonctionnelles.

\section{Le projet}

Une classe de sixième souhaite mettre en valeur une partie du fonds de livres du C.D.I. II s'agit d'inciter les autres à lire, de communiquer ses intérêts, ses découvertes. Pour ce faire, les élèves décident de réaliser un catalogue des livres qu'ils ont lus et particulièrement appréciés. Ce catalogue s'inspirera bien évidemment de ceux que font paraître les éditeurs. Mis en consultation à la bibliothèque, le catalogue des élèves servira de médiateur entre les livres et les lecteurs potentiels.

\section{DÉCOUVERTE DES CATALOGUES}

Pour essayer de répondre à la question posée par la classe « Comment aider quelqu'un à choisir un livre ? ", l'enseignant commence par apporter une quantité importante de catalogues. Les libraires les donnent volontiers, en général les enfants ne les connaissent pas. Le choix se fait en fonction du principe de diversité pour être représentatif de la multiplicité des éditions et des 
collections mais aussi en fonction de la variété des formes et des formats de l'objet-catalogue lui-même. La sélection est construite en tenant compte du niveau auquel on s'adresse : en sixième, L'École des loisirs, le Livre de Poche Jeunesse, Castor Poche, Castermann... fournissent d'excellents exemples.

Dans un premier temps il s'agit d'éveiller la curiosité pour un nouvel objet à lire attrayant que l'on dispense à profusion. Pour faciliter le maniement on peut évoquer d'autres supports proches du quotidien des élèves, qui remplissent la même fonction mais sur des objets différents : La Redoute, Les 3 Suisses, par exemple. Les élèves sont répartis en petits groupes de deux qui dispose chacun d'une dizaine de catalogues très différenciés les uns des autres. Le feuilletage informel est encouragé. Les dessins, les couleurs vives suscitent de l'intérêt. Le format allongé de Casterman, le dépliant en accordéon de Médium Poche à l'École des Loisirs amusent. Dans les tas laissés à l'investigation des groupes, l'enseignant a glissé quelques intrus c'est-à-dire quelques catalogues de livres pour adultes. Ainsi en est-il du catalogue analytique de la Bibliothèque de la Pléiade. Dès la couverture blanche, vierge, il rompt avec les autres. Le papier bible, les fins caractères assez serrés, la galerie de portraits d'auteurs en noir et gris marquent la différence.

Chaque groupe extrait du tas dont il dispose deux catalogues choisis pour leur coté attractif, leur pouvoir incitatif à vagabonder à travers les lectures qu'ils proposent. Le choix doit conduire aussi à dégager deux supports qui pourraient servir de "modèle » au catalogue que la classe veut réaliser. Les intrus jouent un rôle dissuasif : ils sont jugés austères, difficiles à lire, pas assez colorés et illustrés. Les Editions de Minuit comme la Pléiade sont écrits trop petit. Le catalogue 10/18 présente bien des couvertures en couleurs mais ne propose qu'une liste des titres classée par nom d'auteurs sans autre information. Sont préférés le Seuil/Jeunesse aux illustrations éclatantes, les Éditions Milan dont les pages noires mettent en valeur les livres, Bayard Éditions aux nombreux dessins pleine page. Pastel chez l'École des Loisirs séduit avec son format carré et sa sélection d'albums pour les petits jusqu'aux lecteurs confirmés.

Ce libre parcours permet d'opérer une premier tri qui va déboucher sur un travail plus approfondi de lecture et d'analyse.

\section{La grille de lecture}

Chaque groupe a donc à sa disposition deux catalogues qu'il va étudier de façon plus précise. Il s'agit de mener à bien une activité d'observation et d'exploration avec un projet de lecture clairement défini : lire un catalogue et voir comment il est fait pour pouvoir ensuite en réaliser un.

Afin de guider les élèves dans leur lecture, il leur est proposé un ensemble de questions qui permet de dégager trois niveaux distincts d'appropriation de ce nouveau support.

1. L'organisation générale: Quelles sont les différentes parties qui composent le catalogue ? Enumérez-les en relevant les titres qui leur sont donnés. Comment sont regroupés les livres (ordre alphabétique, générique, thématique...) ? Relevez les différents modes de classement.

2. La page : Comment est réalisée la mise en page ? Comment sont disposés les textes (colonnes, pleine page...) ? Quels sont les types de caractères choisis pour les titres, les textes (grosseurs, couleurs...) ? 
Quelles sont les illustrations utilisées et où se trouvent-elles ?

3. Le document: Quelles sont les différentes informations que donne chaque catalogue sur un livre ? Listez-les.

Pendant ce travail de recherche, les élèves peuvent à tout moment demander des explications à l'enseignant qui est là en tant que personne ressource. Les savoirs sur les livres peuvent être très fortement différenciés : certains ont, dès la maternelle, fréquenté les marmothèques et ont une plus grande familiarité avec les livres, d'autres plus démunis ont besoin d'être soutenus. Le recours aux groupes hétérogènes qui fait jouer le conflit socio-cognitif est une première prise en compte de cette diversité.

La mise en commun qui suit cette première lecture à deux et fait intervenir aussi bien les explicitations de l'enseignant que celles des pairs est une nouvelle prise en compte de la différenciation des pratiques culturelles des enfants.

\section{La mise en commun}

Chaque groupe propose le résultat de ses recherches. Le corpus étant extrêmement varié d'un groupe à l'autre, les remarques vont être diversifiées. Les informations sont consignées sur un panneau, ce qui permet de cerner plus finement l'objet catalogue et son rôle. II est possible de repérer des invariants comme la collection, le titre, le nom de l'auteur par exemple. Mais aussi des variantes. Très souvent la première de couverture est donnée ainsi qu'un texte de présentation, le format, le prix, le nom de l'illustrateur... Parfois une notice biographique assortie d'un portrait fait découvrir l'écrivain, des mentions du type nombre de pages, relié, pelliculé mettent en valeur l'aspect matériel du livre.

Toutes ces informations peuvent s'organiser dans un tableau très simple du genre de celui-ci :

Dans un catalogue, je peux trouver :

\begin{tabular}{l|l|l|l} 
& Toujours & Souvent & Parfois \\
\hline $\begin{array}{l}\text { Organisation } \\
\text { générale }\end{array}$ & ordre alphabétique, \\
$\ldots$ & $\begin{array}{l}\text { par genres, } \\
\text { thèmes, } \\
\text { public visé, ... }\end{array}$ & $\begin{array}{l}\text { par auteurs, } \\
\ldots\end{array}$ \\
\hline $\begin{array}{l}\text { Mise en } \\
\text { page }\end{array}$ & $\begin{array}{l}\text { Titre en gros } \\
\text { caractères }, \ldots\end{array}$ & $\begin{array}{l}\text { en colonnes, } \\
\text { couleurs, ... }\end{array}$ & italiques, ... \\
\hline $\begin{array}{l}\text { A propos } \\
\text { d'un livre }\end{array}$ & $\begin{array}{l}\text { le titre, } \\
\text { l'auteur, ... }\end{array}$ & le format, ... & $\begin{array}{l}\text { nombre de pages, } \\
\text { pelliculé, ... }\end{array}$ \\
\hline
\end{tabular}

L'élaboration du tableau est l'occasion de confronter les différentes stratégies développées par les éditeurs mais aussi de répertorier les possibles afin de pouvoir faire son choix par la suite pour construire son propre catalogue. 


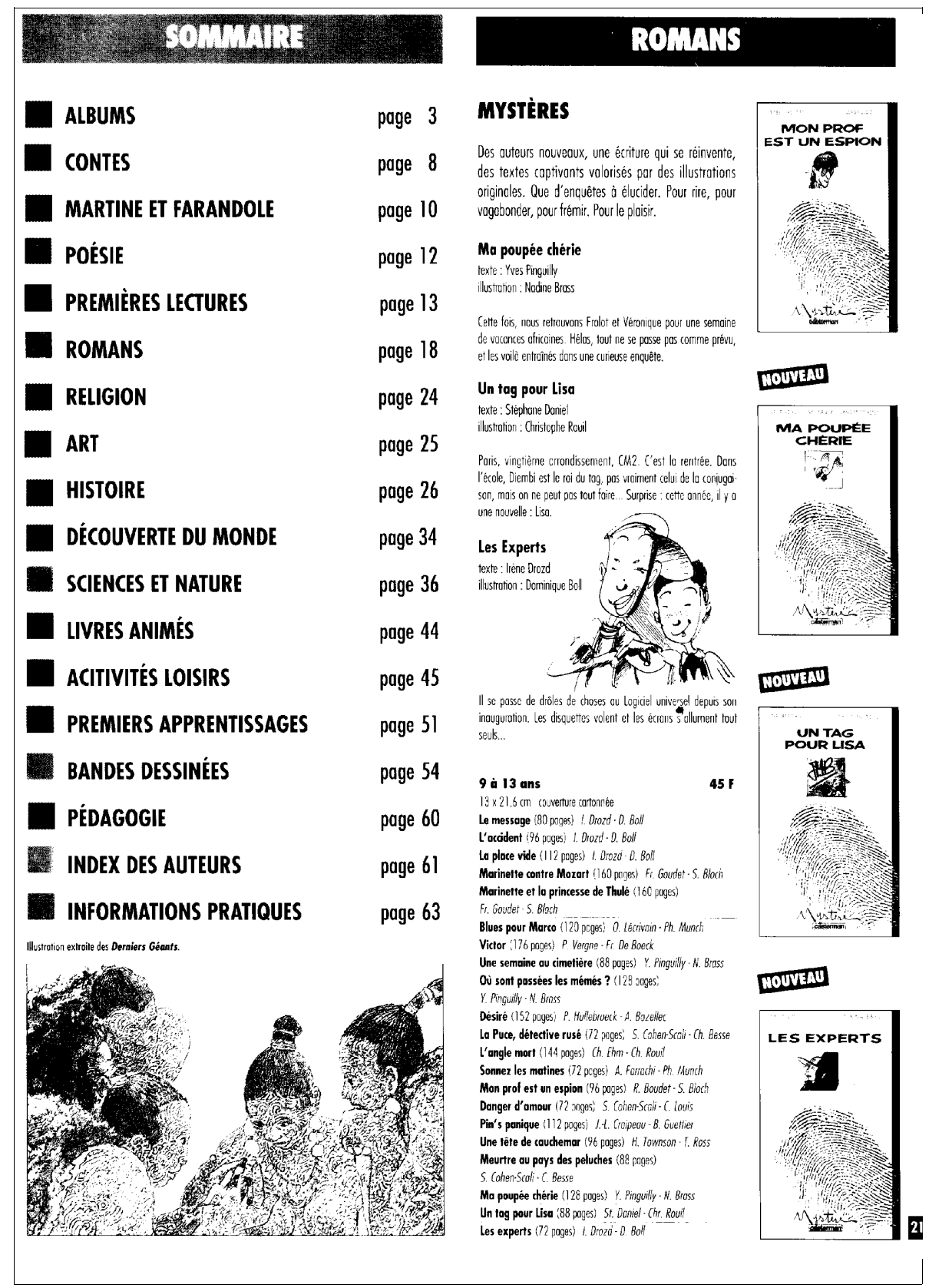

Casterman Jeunesse 


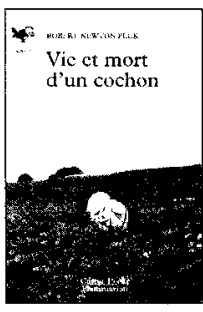

320 Vie et mort d'un cochon Rubert-Newton Peck

raduit de Tangh

par M.-A. Revellat

I.P2 2157

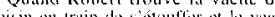
vos min trath de êtonter et de ve:

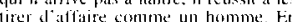

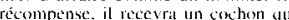
sera sid griande richesse. Mais, dans une ferme si pauve, peut-bu gardet une bélé sealemernl pour le plaisir"

\section{L'enfant multiple}

Andrée Chedid

Calcgoric 5 iscoion) FP 2100

Marylle par la violence el la moz Omar - Jo qui découvre Palris. reven

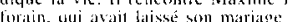

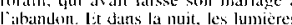
"allument. la messique éclate, la lế commence. I enfant da mitheur deviente messiater de lia jusic: Octogone de chếne 1991
322 Sauvé par les éléphants Hilary Ruben.

raduit de l'anglais par I. Paves

Catcintict

$1 \mathrm{p}=|6|$ illisals. konyeh, se fill allaquet wher son troupeall. Cache. son cousin

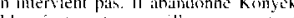
bese el rentre su tillage racontant

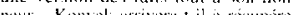

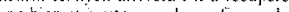
iाius?

323 Drôle de Noël :

Wolf Spillner

Wallemand

par F. Mathieu

Ifliguric 4 IP 21.36

Lid arand-mice de Hane a pour seule compitynic un pigeun L animal vil dians a cumsine, le que sa belle-lille ne pelut pis supporter. Le four de Novis Hans voil sa mere le pigeon mort it matin. Fille pretend livstir the sitns le volitilir. Hatrs tente de comprendre... Prix allemand de la J ittérature pour ta Jeunesse 1991

324 La famille dispersée Le Train des orphelins

Joan Lowery-Nixon,
tratuit de l'anglais (Etats-Unis par A.-M. Chapouton

Cittiguric 7

IP? 2162

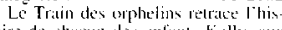
toire de chateun dex enlianls Kelly. ilux

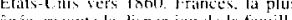
dyece laconte la dispersion de la famille

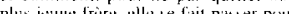

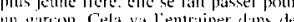
Pring

P'rix des Incorruptibles 1991
Castor Poche

Flammarion

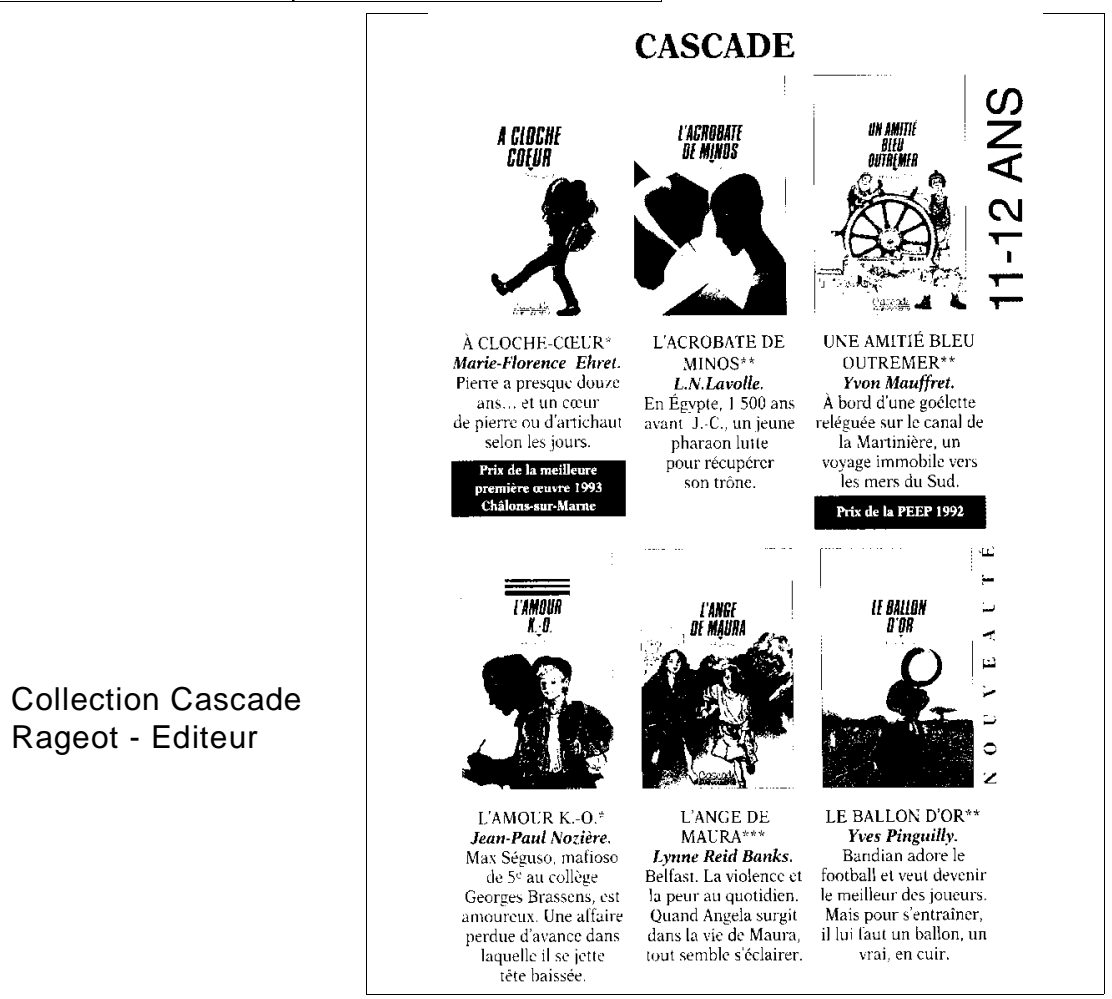


C'est également un temps d'explicitation ou de remise au clair de certaines notions, de certains savoirs. « $27,5 \mathrm{~cm} \times 20,5 \mathrm{~cm}$ » peut rester longtemps une énigme pour de jeunes lecteurs. Quelques téméraires suggérent, tout en n'y croyant pas, qu'on pourrait peut-être faire la multiplication. Le terme « format » est loin d'être partagé.

\section{ANALYSE DES CATALOGUES}

Le matériau est riche, la collecte est donc abondante. Quand la classe inventorie les façons d'organiser un catalogue, elle découvre que les livres ne sont pas seulement rangés par ordre alphabétique mais que bien souvent ce classement se combine avec un regroupement par genres et/ou par thèmes. $A$ cela peut même s'ajouter, par exemple, chez Rageot-Editeur dans la collection Cascade des entrées par public visé (7-8 ans, 9-10 ans, 11-12 ans). Pour faciliter les déplacements dans le catalogue et rendre plus lisible le dispositif, il est parfois proposé un code chromatique qui guide l'usager. Ainsi Casterman Jeunesse désigne les contes en violet, la poésie en bleu, les romans en vert... Les classements ne sont pas homogènes et ce même catalogue utilise également des catégories très disparates où interviennent la spécificité du support (ex. : bandes dessinées), une série porteuse (ex. : Martine), une particularité éditoriale (ex. : livres animés). Cet éclectisme ne se rencontre pas seulement dans les catalogues, les bibliothèques, les librairies peuvent présenter une offre très ouverte. II n'est donc pas inintéressant de faire réfléchir les élèves aux différents modes de classements pour les familiariser avec l'ordre du monde des livres.

Les catalogues d'éditeurs ont aussi un paratexte développé. Bien souvent ils s'ouvrent par un sommaire ou se ferment par une table des matières. Les élèves se demandent si les deux termes sont équivalents et s'ils recouvrent la même réalité. Ils ne tardent pas à s'apercevoir que la place détermine l'appellation : en ouverture, on dit toujours sommaire et en fermeture, table des matières. Mais la différence de sens est plus difficile à cerner et les définitions du dictionnaire ne sont pas toujours respectées dans le fonctionnement réel des catalogues.

Le recours à l'index est aussi une caractéristique fonctionnelle de ce type de support. C'est une médiation extrêmement utile, quand on sait s'en servir, pour trouver plus rapidement une information précise. Les plus fréquents sont les index des titres et les index des auteurs. Certains catalogues comme par exemple celui du Livre de Poche Jeunesse offre des index à profusion : par titre, par âge, par genre, par thème.

Plus rare, une introduction dans les premières pages présente la collection. Elle peut mettre en valeur le contenu des livres. " A ses lecteurs de 7 à 15 ans, Le Livre de Poche Jeunesse propose 300 titres : une grande variété de genres et de thèmes adaptés à chaque âge. Pour susciter la curiosité, le rire et l'émotion, le roman se fait historique, fantastique, policier ou récit d'aventures... » L'aspect matériel du support peut être aussi valorisé : « Un Lecture Junior s'ouvre sans effort, étale sur un papier souple de belles pages foisonnantes d'illustrations en couleurs... "

Autant de stratégies différentes qui permettent aux élèves de se construire progressivement des représentations plus réalistes d'un discours d'incitation à la lecture et de mieux baliser le cheminement documentaire. 
La recherche sur la mise en page est aussi d'un grand apport. Les livres peuvent être présentés en colonnes comme chez Castor Poche Flammarion. Mais ils peuvent aussi être offerts horizontalement : chez Cascade, la page est divisée en deux parties traitant de trois livres chacune. Grasset-Jeunesse consacre une page aux «Contes d'ailleurs et d'autre part » de Pierre Gripari de façon en mettre en valeur un titre particulier.

Tous les catalogues sont en général très illustrés. La première de couverture est omni présente mais également certaines illustrations des pages intérieures. Les couleurs vives dominent et les élèves ont éliminé de leur corpus le catalogue Gallimard Jeunesse parce qu'il ne comportait que quelques dessins en noir et blanc.

Outre l'aspect attractif des illustrations, la typographie et le jeu sur la grosseur des caractères participent également des stratégies de présentation des livres. Les grandes lettres, les petites, les caractères gras, les italiques, les couleurs ont une signification qu'il faut apprendre à décoder.

Le travail sur la présentation d'un livre met les élèves en situation de se familiariser plus finement avec les mots et les façons de dire du lire. II s'agit en effet de faire découvrir aux jeunes lecteurs que les livres se parlent dans des discours spécifiques, à l'aide d'un vocabulaire particulier.

Le catalogue est parfaitement adapté pour concrétiser les notions d'édition et de collection. L'École des Loisirs est un excellent support. En effet cette maison varie ses catalogues en fonction de ses collections. Il est facile d'exposer sur une table côte à côte Mouche (romans pour jeunes lecteurs), Neuf ( romans pour lecteurs confirmés), Médium (romans pour adolescents), Archimède (albums documentaires), Pastel (albums pour les petits et les plus grands), Lutin Poche (albums « classiques » pour les petits). Les caractéristiques d'une collection peuvent être dégagées, une réflexion peut être engagée sur la multiplication des collections et l'intérêt d'une telle stratégie pour un éditeur.

D'autres termes suscitent échanges et interrogations. "Illustrateur » ne pose pas de problème de compréhension mais son rôle est flou, beaucoup d'élèves sont persuadés que c'est l'auteur qui dessine et qu'il ne peut en être autrement. «Traduit de » montre que nombre de livres appartenant à la littérature de jeunesse sont étrangers. Pour certains, c'est une découverte : stupeur de deux sixièmes qui s'aperçoivent que Le Club des Cinq est un roman écrit à l'origine en anglais! «Adapté par » introduit plutôt la suspicion chez les jeunes lecteurs, peut-être au nom d'une certaine sacralité du texte et de l'auteur. Ce type de travail permet justement de discuter ensemble des raisons idéologiques, économiques ou pédagogiques de ces adaptations. Le prix, quand il est indiqué, permet de faire une incursion dans l'univers marchand et d'apprendre à évaluer la valeur de l'objet sur le marché des échanges.

La présentation du livre comprend bien souvent un texte qui accompagne les informations ponctuelles. Les catalogues visant un jeune lectorat, les textes centrent davantage sur la fiction que sur la narration. Les élèves les appellent des résumés mais c'est une appellation qui peut prêter à confusion. Une lecture un peu attentive montre que ce ne sont pas des résumés au sens scolaire du terme. II n'est pas question de raconter l'histoire dans sa totalité sinon l'effet incitatif est rompu. Ces textes au contraire doivent constituer une amorce à la lecture et sont construits de façon assez stéréotypée. Ils s'ouvrent par une ou deux phrases qui condensent le début de l'histoire ou état initial. La phrase suivante met en valeur l'événement perturbateur, pivot autour duquel tourne tout le 
récit. Une ou deux actions permettent de faire monter le suspense et de préparer la fermeture. Les points de suspension sont le procédé le plus fréquemment utilisé pour clore ce genre d'écrit : ils miment l'inachevé du texte et ouvrent sur la lecture future. Le point d'interrogation est également très employé, il donne aussi au texte cette fin ouverte caractéristique de ce type de discours. Le point d'exclamation ponctuant une relance sur le caractère inouï de l'histoire se rencontre assez souvent. Le point seul peut terminer une phrase qui induit des possibles narratifs (ex. : « Et cette rencontre va changer beaucoup de choses. $")$.

Voici un exemple de texte de présentation assez représentatif de ce que l'on peut lire. Il est extrait du catalogue Castor Poche Flammarion.

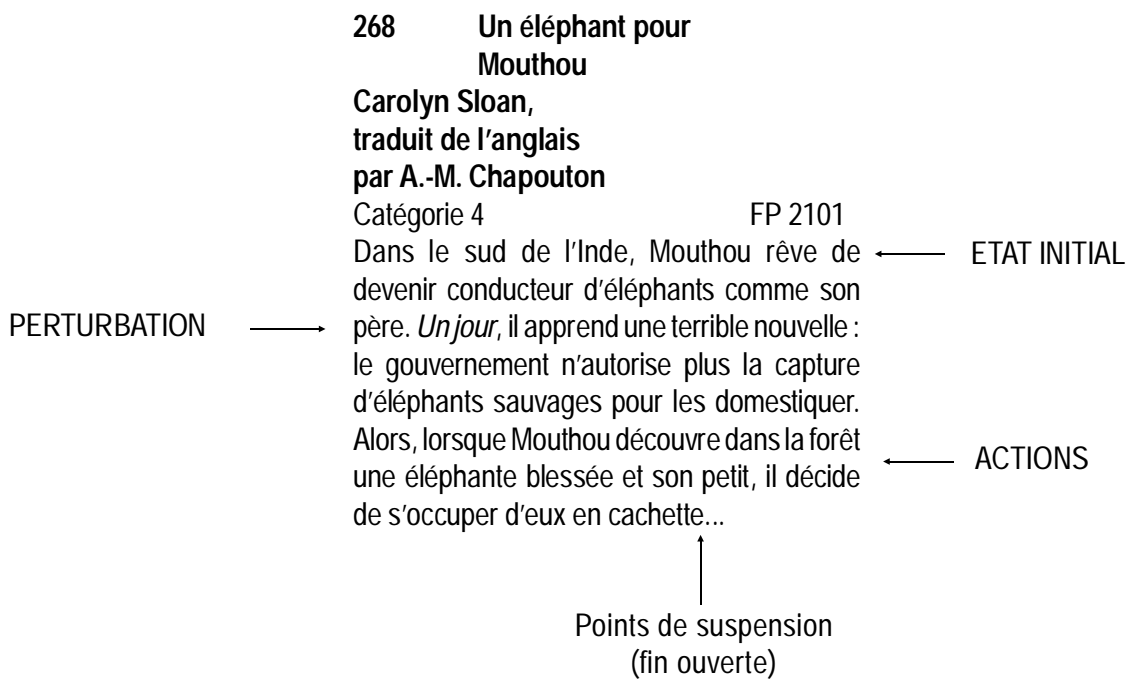

La présentation d'un livre s'accompagne parfois de la notice biographique de l'auteur à laquelle est joint un portrait photographique. Les jeunes lecteurs de sixième sont toujours friands de ce genre d'informations : ils aiment voir à quoi ressemble quelqu'un qui écrit des livres. D'ailleurs les éditeurs pour la jeunesse essaient de rendre l'écrivain plus proche, plus familier en donnant des renseignements qui ont trait à la vie quotidienne. "Jean-Philippe Arrou-Vignod est né en 1958, il a cinq frères, il est papa de deux enfants et il est professeur dans un collège de Reuil-Malmaison. II adore jouer aux échecs... " (Catalogue Neuf, École des Loisirs). En cherchant à s'approprier ces supports, les élèves travaillent aussi leurs représentations des écrivains et font évoluer les images stéréotypées qu'ils sollicitent habituellement.

La lecture construite des catalogues est une activité riche qui fait entrer, de façon concrète, les élèves dans la pratique réelle des livres et des lecteurs. Elle éveille leur curiosité, elle les met en situation de se poser des questions, de construire un premier état de réponses qui les autorise ensuite à continuer leurs investigations grâce à l'assurance que donne un début de familiarité dans un domaine donné. 


\section{LA PRODUCTION DU CATALOGUE CLASSE}

Après l'activité de lecture vient le temps de la réalisation du catalogue. Les élèves doivent produire à leur tour un objet capable d'inciter des pairs à lire. Chacun choisit un livre qu'il a déjà lu et qu'il a particulièrement aimé. Ce livre appartient au fonds du C.D.I. du collège. Aucune contrainte de genre ni de support n'est donnée, pourtant sont largement privilégiés les textes de fiction même si les documentaires sont présents à la bibliothèque. Un projet plus systématique sur les livres informatifs devra être mené ultérieurement de façon à familiariser les lecteurs avec la spécificité de ces écrits.

II ne faut pas seulement choisir le livre sur lequel on va travailler mais décider collectivement de la forme que va prendre le catalogue. Un travail important de planification est à réaliser. La classe doit se mettre d'accord sur l'organisation générale, la mise en page et la présentation du livre. Retour est fait au tableau récapitulatif réalisé lors de l'activité de lecture, parfois même certains catalogues sont repris pour préciser les choix. De toute façon on aboutit à une liste des différentes entrées à prendre en charge qui est un peu comme le premier état du sommaire. Il est décidé de regrouper les livres selon les genres, à chaque genre correspondant un bandeau de couleur différente. On prend également la décision d'une présentation pleine page. On réalise donc ensemble la maquette d'une page qui servira ensuite de matrice à chaque scripteur.

Un exemple :

$\begin{array}{cl}\text { 1) Illustration } & \text { titre } \\ \left.\text { (1 } 1^{\text {re }} \text { de couverture }\right) & \text { auteur } \\ & \text { éditions } \\ & \text { collection } \\ & \text { format } \\ & \text { nombre de pages } \\ & \text { traduit de } \\ & \text { texte intégral } \\ & \text { illustrations } \\ \text { public } v \text { isé }\end{array}$

La mise en texte est un travail individuel. II s'agit pour chaque élève de fournir les informations sélectionnées concernant son livre et de rédiger un résumé ouvert. Claire présente ainsi Le professeur a disparu de Jean-Philippe ArrouVignod.

"La ville avait organisé un concours d'histoire-géo. Les trois gagnants du voyage à Venise étaient Pierre-Paul de Culbert dit P.P.Cul-Vert, Rémi Pharamon et Mathilde Blondin. Rémi, lui, n'aurait pas du gagner le concours. C'était son meilleur copain, Philibert, qui devait l'emporter. Mais l'oncle de Rémi, agent de mairie, avait pu avoir les sujets à l'avance. Pourtant pour un peu il n'y arrivait pas ! Dans le train pour l'Italie, le professeur qui accompagnait les enfants avait disparu et Mathilde avait aperçu un homme bizarre. Si Rémi avait su ce qui allait arriver, il ne serait peut-être jamais parti... »

Claire décide de mettre en valeur les noms des personnages en fonction du public visé. Elle fait l'hypothèse que le jeu sur le surnom, l'évocation de Pharaon dans Pharamon, la personnalisation fille (Mathilde), garçon (Philibert) seront 
autant d'accroches à la lecture pour des pairs à convaincre. Elle signale la débrouillardise de Rémi pour gagner le concours car cela rend sympathique le personnage. Elle s'est demandée si elle devait préciser davantage les circonstances de la disparition du professeur et a finalement choisi l'expression " homme bizarre » qui suggére des possibles narratifs variés. Enfin elle a opté pour les points de suspension qui laissent la phrase de clôture ouverte et accentuent sa dimension incitative.

Une fois le travail de mise en texte réalisé, la fabrication du catalogue avec illustrations intervient. II s'agit de reproductions en réduction de la première de couverture du livre choisi, de collages d'illustrations de pages intérieures. Un sommaire organisé de façon générique avec un code de couleurs est placé en tête du catalogue, un index des titres et un index des auteurs en fin. Les pages numérotées, sont reliées par une spirale et recouvertes d'une couverture abondamment décorée. Le catalogue, déposé au C.D.I., est consultable par tous les élèves. II peut même faire l'objet d'un échange entre classes en fonction des travaux et des projets.

\section{AUTRES PISTES, PROLONGEMENTS}

Le travail présenté ici peut être l'objet de nombreuses variantes et adaptations. En effet des modifications sont apportées en fonction du niveau ou des situations d'utilisation des catalogues.

En troisième, le catalogue produit par les élèves peut s'ouvrir par une introduction générale de type argumentatif qui, sur le modèle des présentations éditoriales, justifie pour le public visé les livres choisis quant aux auteurs, aux genres, aux thèmes, aux collections. La sélection des arguments, l'organisation et la progression de l'argumentation participent à l'élaboration et à l'appropriation d'un système de valeurs qui aide à construire la croyance dans l'intérêt du livre.

Les catalogues d'éditeurs peuvent s'utiliser au sein de la classe pour choisir les livres à étudier par exemple dans le cadre de la lecture suivie ; en groupes, pour compléter le fonds du C.D.I. dans un dispositif de collaboration avec le documentaliste.

Les catalogues peuvent encore être sollicités pour faire des repérages comparatifs entre les différentes collections et éditions d'un même ouvrage et apprendre ainsi à choisir l'outil le mieux adapté au niveau du lecteur et à la tâche qu'il a à réaliser. En sixième, un projet d'écriture de fables nécessite par exemple la constitution d'un corpus. Les fables de La Fontaine proposées en Livre de Poche Jeunesse sont rapidement sélectionnées : les indications fournies à propos des illustrations, Grandville et Morgan ; la mention « Fables choisies " sont convaincantes. Est retenu également Folio Junior chez Gallimard qui propose aussi un choix de fables mais cette fois avec des illustrations de Gustave Doré. Par contre Le Livre de Poche adulte signale un La Fontaine intitulé Fables pour tout renseignement, les jeunes lecteurs en concluent qu'il n'y a pas d'illustration et font l'hypothèse d'un nombre de textes plus important, d'un balisage inexistant.

Autre support utilisable dans la classe, les présentations de livres que l'on trouve dans les revues destinées à la jeunesse. Un magazine peut servir d'exemple, Je Bouquine et son dossier littéraire. Dans chaque numéro, parmi 
des articles variés reprenant toujours les mêmes entrées, actualité culturelle, humour, histoire vécue, fiction complète racontée en une trentaine de pages, se trouve la présentation, toujours très illustrée, d'un livre qui appartient au patrimoine cultivé des lectures adolescentes. Cette présentation s'ouvre par une accroche en bande dessinée : le début du roman est racontée en images. Suit une vie de l'auteur mise en page comme un article de presse. Le dossier se clôt par une fiche de lecture. Cette fiche, rénovée par rapport aux traditionnelles fiches de lecture, constitue une excellente matrice de travail. La situation de communication est dynamique car il s'agit, comme dans le catalogue, d'inciter des pairs à la lecture. Seul le support varie. Cette fois l'activité de production d'écrit sera plus importante.

En effet la fiche comporte un résumé ouvert sur le modèle des résumés de catalogue mais beaucoup plus développé, chaque étape est enrichie. Si l'on prend l'exemple de La Potion Magique de Georges Bouillon (Je Bouquine, no 81, novembre 1990, p. 88), après les points de suspension signalant la fin inachevée de l'histoire, on note la présence d'un paragraphe incitatif, ultime accroche, dont le rôle est de mimer le désir du lecteur. "Ce serait dommage d'en révéler davantage. Si vous voulez connaître la suite de cette histoire insensée et hilarante, précipitez-vous sur La Potion Magique de Georges Bouillon sans plus tarder! »

Un extrait donne ensuite un aperçu concret du texte. Les sixièmes ont du mal à distinguer extrait et résumé. II faut des va-et-vient nombreux entre des résumés réalisés à haute voix par les élèves sur un texte qu'ils connaissent et des lectures faites livre ouvert en respectant le mot à mot de l'histoire pour que les deux notions commencent à se différencier. Une réflexion sur le choix de l'extrait ne manque pas d'intérêt. En étudiant le travail fait sur Georges Bouillon on s'aperçoit que l'extrait prolonge le suspense lancé par le résumé ouvert : les effets de la potion sont explicités, la figure de Grandma est magnifiée mais aucun des possibles narratifs n'a pas été refermé. De plus l'extrait doit être suffisamment représentatif pour suggérer la façon d'écrire de l'auteur, la tonalité du texte. " - Branle-bas ! criait-Grandma. Là où je passe l'herbe trépasse ! Hors de mon chemin, misérables crapoussins! Sinon je vous écrabouille! ». Ces phrases rendent bien compte de l'humour de l'histoire et de son caractère loufoque et hilarant.

La fiche se poursuit avec des indications bibliographiques sur l'auteur concerné. Dans l'exemple précis plusieurs ouvrages de Roald Dahl sont cités, classés par genre et par public visé. Avec les élèves, il faut relever les informations retenues (titres, éditions, collections), les présentations rapides et incitatives des ouvrages... En situation de production, pour réaliser un travail similaire, il faudra faire des recherches au C.D.I. de son établissement, dans les catalogues d'éditeurs pour construire un bibliographie crédible.

Enfin une galerie des principaux personnages clôt le dossier. Un médaillon sous forme de dessin humoristique sert d'en-tête à quelques phrases qui peignent à grands traits les héros. Les constructions nominales ("Une méchante vieille sorcière. "), les adjectifs qualificatifs sont utilisés dans cette caractérisation rapide.

Faire réaliser de tels dossiers par les élèves permet aussi de travailler de façon active les appropriations culturelles. Présentées sous forme de petits 
fascicules aux dessins colorés (reproductions de premières de couverture, d'illustrations de pages intérieures), ces médiations lecturales circulent entre les lecteurs, offertes sur une table de lecture au C.D.I., accompagnées du livre qu'elles présentent.

La liste des activités possibles est loin d'être close. A tous les niveaux du collège peut être proposée la réalisation d'un catalogue sur un auteur. Les élèves, placés en situation de recherche active, se construisent ainsi progressivement une culture littéraire et des habiletés lettrées. Selon que les écrivains appartiennent ou non au patrimoine légitime, les éditions et les collections dans lesquelles paraissent leurs ouvrages sont plus ou moins nombreuses. "Poil de Carotte " de Jules Renard ne compte pas moins d'une vingtaine de parutions différentes, à l'inverse «La Sixième » de Susie Morgenstern ne se trouve qu'à l'École des Loisirs.

Le catalogue d'auteur peut s'inscrire également dans le cadre d'une rencontre avec un écrivain. II constitue un travail préparatoire de prise de connaissance et de partage de lectures autour d'une œuvre particulière.

Le catalogue peut mettre en valeur un genre comme le policier, la bande dessinée... Là encore il s'agit d'apprendre à dégager et à construire les repères propres aux éditions et collections spécialisées dans ce type d'ouvrages. La B.D. permet d'explorer la diversité des graphismes, des styles : Hergé n'est pas Hugo Pratt!

On peut aussi travailler sur un thème précis, les animaux ou la mer par exemple, sur un ouvrage particulier. Le Colonel Chabert, Le Hussard sur le Toit sont autant de textes qui bénéficient non seulement d'une bibliographie riche en ce qui concerne le récit lui-même, les analyses critiques mais aussi d'une filmographie et d'une discographie contemporaines qui ouvrent la recherche à d'autres types de supports.

Le catalogue peut encore concerner un héros qu'il est facile de choisir en fonction de l'âge et des intérêts des élèves. Pour les plus jeunes on pense à Astérix, à Tintin mais également au Kamo de Daniel Pennac, au Sans Atout de Boileau-Narcejac, à l'intrépide William de Richmal Crompton, à l'attachant P.P.Culvert de J.-P. Arrou-Vignod... Pour les plus grands, il y a Hercule Poirot, Sherlock Holmes, Arsène Lupin et Nils Hazard, le savant étruscologue et détective imaginé par Marie-Aude Murail. Le lecteur peut aussi se transformer en détective et prendre en filature son héros dans des corpus de textes classiques: suivre Rastignac dans la Comédie Humaine, Octave Mouret dans les RougonMacquart. II s'agit ici de s'appuyer sur des solidarités liseuses, de se plonger à plusieurs (groupes) dans des textes qui peuvent effrayer certains lecteurs d'avoir un projet de lecture qui autorise un parcours sélectif, de repérer les passages qui mentionnent le nom du héros choisi de façon à comprendre le rôle qu'il joue dans l'histoire.

Chaque élève peut enfin, tout au long de l'année, réaliser le catalogue de ses lectures. Dans ce cas, l'appréciation portée par le lecteur pourra être positive ou négative. II faudra alors apprendre à étayer son jugement et pour cela se référer aux rubriques d'analyses de livres de certains journaux ou magazines afin de se construire un discours approprié. 
A vous lecteur, mon semblable, de continuer ce catalogue des activités-catalogue...

Le travail présenté peut donc se justifier à plus d'un titre.

- Il offre une situation de recherche active qui propose une entrée nouvelle dans les livres en allant à la rencontre de l'offre éditoriale.

- Il fait intervenir la lecture et l'écriture : tout au long du projet, les élèves lisent des catalogues, des livres, écrivent des résumés ouverts, des appréciations, des notices bibliographiques, des fiches techniques, échangent et parlent de leurs lectures-écritures.

- Il familiarise les élèves avec des médiations qui structurent l'univers des pratiques du livre.

- Il favorise ainsi l'accès à la culture littéraire par une fréquentation plus intense des références aux œuvres et aux discours sur ces œuvres.

- II permet enfin la construction d'un rapport lettré au livre par l'acquisition d'un vocabulaire spécifique et de classements précis, par l'élaboration d'un discours critique, par la compréhension progressive des enjeux et des valeurs liés à la pratique de la lecture. 